\title{
EDITORIAL
}

DOI: http://dx.doi.org/10.19053/22160159.v7.n15.2016.5720

\section{SOBRE LA ESCRITURA COMO EXPERIENCIA}

Escribir se ha convertido en una de las prácticas más solicitadas y promocionadas en la actualidad. En el mundo académico no solo como una posibilidad de comunicación de los hallazgos de los procesos y proyectos de investigación, sino como una forma de mercantilización de los saberes y circulación de discursos impersonales que se han constreñido a formatos, condiciones específicas y normas para seguir paso a paso. Qué pena que este arte de la vida, la existencia y la razón haya sido colonizado y utilizado por los centros de poder y de saber, que en favor del capital y de sus flujos e intensidades la han colocado, a la escritura, como una esclava, para legitimar la circulación y apropiación de ciertos estatutos teóricos hegemónicos, a los intelectuales y académicos, a su vez como los medios para conseguir estos fines a cambio de salarios, puntos, índices de citación y clasificación en los rankings propios de las diferentes manifestaciones de la ciencia.

Esta pequeña introducción un tanto apocalíptica y paradójica sobre la escritura, y más en la editorial de una revista Indizada y reconocida, como Praxis \& Saber, nos permite abordar algunos aspectos que considero debemos como académicos y productores de saber recordar y reactualizar cada vez que emprendemos la dura, extenuante y difícil arte de la escritura. Son cuatro aspectos que se presentan: la escritura como experiencia, ensayo y áskesis.

Se resalta en primera instancia que la escritura se inscribe en el ámbito de la experiencia, es decir, que escribir es experimentar, jugar con el pensamiento producir o recrear conceptos, actualizarlos e intentar producir en uno mismo y en los otros efectos de transformación, nuevas formas de mirada y posibilidades de acción. En este sentido, la experiencia se concentra en producir a través de la escritura desplazamientos, rupturas y alertas en 
palabras de Foucault (2013), reconociendo la escritura de Nietzsche, Bataille y Blanchot, afirma que «...la experiencia consiste en tratar de alcanzar cierto punto de la vida que esté lo más cerca posible de lo invivible. Lo que se necesita es el máximo de intensidad, y al mismo tiempo de imposibilidad» (p.35). Este sitio de la experiencia escritural pretende «arrancar al sujeto de sí mismo, procurar que ya no sea el mismo». El sujeto escritor se desubjetiva y es otro, radicalmente otro y ya no vuelve a ser el mismo.

Un segundo aspecto es el ensayo, o mejor, la escritura como ensayo, representa uno de esos mojones claves para para la constitución de sujetos, es la posibilidad de ejercitar, con relación al ejercicio de escribir, el pensamiento, hacer ejercicio del mismo y construir posibilidades de creación, de modificación de sí mismo en regiones de verdad tan instrumentalizados como los del capitalismo cognitivista contemporáneo. Escribir ensayando y ensayar escribiendo puede significar no solo formas novedosas de comprender los campos teóricos comprometidos en la investigación educativa y pedagógica, sino lo más importante, la transformación de los sujetos involucrados.

La tercera invitación sobre la escritura es la áskesis, o para decirlo en otra forma la escritura es áskesis, ejercicio de sí, sobre sí mismo y por ende del pensamiento, aleja los fantasmas de soledad, «el hecho de obligarse a escribir desempaña las veces de un compañero, suscitando el respeto humano y la vergüenza» (Foucault, 1999, p.289). Este ejercicio implica lectura, meditación, relectura para asimilar los que se piensa y que pueda servir para uno mismo en forma de recuerdo y para los otros. La escritura se asocia a dos modos diferente del pensar una que se puede afirmar lineal que va de la meditación a la escritura y de la escritura el entrenamiento para lo real, trabajo del pensamiento para recrear y reconstruir la realidad. El otro es circular meditación-relectura-meditación, «la escritura constituye una etapa esencial en el proceso al que tiende toda áskesis: a saber, la elaboración de discursos recibidos y reconocidos como verdaderos en principias racionales de acción. La escritura como elemento del entrenamiento de sí, tiene, para utilizar una expresión que se encuentra en Plutarco, una funciónn ethopoiética: es un operador de la transformación de la verdad en éthos» (Foucault, 1999, 291).

En este número Praxis \& Saber presenta escrituras convertidas en artículos de investigación que privilegian las relaciones entre didáctica y aprendizaje atravesadas por experiencias investigativas que indagaron por procesos de aula y formas de trabajo concretas en escuelas y universidades. 
El primer grupo de artículos presentan investigaciones referidas a la didáctica y su relación con la nuevas tecnologías de la información y la comunicación, es así como la profesora Nancy Peré, de la Universidad de la República, Uruguay, en un trabajo titulado apuntes para pensar la relación entre innovación, tic y formación pedagógico didáctica, apuestan por un conjunto de puentes reflexivos que relación innovación, tic y formación docente como apuesta desde la didáctica y la pedagógica para incidir en profesores universitarios uruguayos. En el mismo sentido aparece un trabajo titulado efecto del entrenamiento en la autorregulación para el aprendizaje de las matemáticas de los profesores Luis Sanabria Rodríguez, Nilson Valencia Vallejo, Jaime Ibáñez Ibáñez, de la Universidad Pedagógica Nacional, en él encontramos la descripción de la utilización de una estrategia pedagógica en una ambiente B-learning como sistema de autorregulación para el rendimiento académico, utilizando para el análisis de los datos ANCOVA.

Otro grupo de artículos publicados en este fascículo convergen en intervenciones en el aula partir de procesos comunicativos, tecnológicos y de metodología por proyectos. En el trabajo titulado patrones de interacción comunicativa de algunos docentes universitarios de matemáticas. Caso «Fernando», el profesor José Francisco Leguizamón Romero, de la Universidad Pedagógica y Tecnológica de Colombia, presenta los resultados de una investigación en la cual analiza patrones de interacción comunicativa de algunos profesores de matemáticas de la UPTC, a partir de un análisis cualitativo y materializado en una estudio de caso, en el cual se concluye que el tipo de clase del profesor entrevistado es tecnológico-tradicional. Las profesoras Olga Lucía Londoño Palacio, Liccy Catalina Calderón Villafáñez, Paola Lucumí Useda, Martha Alexandra González Casteñada de la Escuela Normal Superior Ubaté, presentan el texto titulado, experiencia innovadora de la estructuración ontológica del conocimiento con docentes y estudiantes, presenta los resultados de un proyecto de investigación que realiza una caracterización sobre el manejo de competencias digitales en grupos de profesores utilizando dispositivos tecnológicos. De igual forma aparece en esta sección un trabajo de los profesora Alexandra Ruíz Martínez, del Instituto Tecnológico y de Estudios Superiores de Monterrey titulado estrategia didáctica para el aprendizaje de los ciclos biogeoquímicos desde la transdisciplinariedad en el cual analiza la implementación de proyectos estudiantiles como estrategia para reestructurar preconcepciones tradicionales de la ciencia, se utiliza para el análisis un modelo mixto. 
El tercer grupo de artículos del actual número de Praxis \& Saber, muestra 4 trabajos en los cuales se relaciona escritura, oralidad, representaciones sociales y estética. El artículo titulado oralidad y escritura: campos de investigación en pedagogía de la lengua materna es una investigación documental en la Maestría en Pedagogía, de la lengua materna de la Universidad Distrital Francisco José de Caldas, para tomar decisiones a propósito de los resultados de la misma. En otro sentido el artículo titulado Actitudes hhacía el amor en relaciones románticas de jóvenes universitarios de los profesores Brenda Liz Rocha Narváez, Claudia Estella Avendaño Parra, Miguel Alfredo Barrios Alonso, Adalgisa Polo, de la Universidad de la Sabana, realizan una caracterización de la tipologías de amor más sobresalientes en las relaciones de pareja en de 310 jóvenes (hombres y mujeres) de la UPTC sede Tunja. En la linea de investigación sobre arte y educación se encuentra el trabajo de Hercilia Aurora Gordo Contreras y Joan Sebastián Igua Muñoz de la Universidad Pedagógica y Tecnológica de Colombia titulado la dimensión empírica de la estética en la educación escolar que pretende establecer las relaciones del contexto periférico escolar en el comportamiento y los factores condicionales dentro de la escuela si potencian o disminuyen la conducta socieoestética. Este proyecto de investigación fue desarrollado en una escuela urbana y otra rural de la Ciudad de Tunja.

En el cierre de este fascículo aparece un grupo de tres articulos que reunen dos tipos de escritura uno está a cargo del profesor Armando Zambrano Leal pedagogo e investigador colombiano que nos presenta un trabajo de investigación, titulado Gobernanza de la escritura: la escisión entre modernidad e hipermodernidad «la cita y el parentesis» que relaciona las formas de escritura en la modernidad y la hipermodernidad, tomando como aspecto de análisis las formas de escribir científicamente en las ciencias Sociales, en este caso la utilización de formas técnicas de la escrutar de artículos y libros.

Y por último aparecen dos artículos artículos de revisión y asumen una manera novedosa de presentar resultados de investigación, se ubican como balance de campos teórico, en esta caso de las relaciones entre filosofía e infancia y el programa filosofía para niños $(\mathrm{FpN})$ en Colombia de las investigadores Paola Andrea Lara, María Teresa Suarez Vaca y Bibiana Alexandra González de la UPTC, y por otra parte para finalizar el fascículo en un artículo titulado ¿qué se escribe sobre investigación y pedagogía? Que realizan una analisis conceptual de la producción académica en las revistas Pedagogía y Saberes y Praxis \& Saber, producto de un proyecto de investigación financiado por el Centro 
de investigación de la Universidad Pedagógica Nacional CIUP-UPN y del cual son autoras las profesoras Ana Cristina León Palencia, Yeidy Alexandra Gil Pinto, Erica Yuliana Cárdenas Vera, María de los Ángeles García Zúñiga, Greizzy Eliana López Reyes.

Los invitamos a todos y todas disfrutar la lectura de estos interesantes trabajos que reflejan formas diversas de escritura e investigación, de igual forma queremos compartir con ustedes que Praxis \& Saber, por el gran volumen de artículos y la aceptación en la comunidad académica en educación y pedagogía, a partir del año 2017 cambia su periodicidad a cuatrimestral. En la actualidad en el Open Journal System (página web) serán publicados (3 fascículos al año), para poder cumplir con la demanda.

Así mismo, la revista el 12 de enero del 2017 ingreso a la Sistema de Información Científica Redalyc, Red de Revista Científicas de América Latina y el Caribe, España y Portugal; cumpliendo satisfactoriamente con los criterios de calidad editorial considerados por esta prestigiosa base de datos, en la circulación y divulgación a nivel nacional e internacional de los artículos, para la consulta de investigadores.

\section{Referencias}

FOUCAULT, M. (1999). La escritura. En Foucault, M. Estética, Ética y Hermenéutica. Obras Esenciales Volumen I. Barcelona. España. Paidós. FOUCAULT, M. (2010) Como nace un libro experiencia. En Trombadori, D. Conversaciones con Foucault. Buenos Aires. Argentina. Amorrortu FOUCAULT, M. (2012) Un peligro que seduce. Valladolid. España. Cuatro ediciones.

\footnotetext{
Oscar Pulido Cortés Leonor Gómez Gómer.
} 